\title{
Interleukin-8 (IL-8) seen as an actor with several roles in the tumor development
}

\author{
Manuela González ${ }^{* *}$ and Carlos Alfaro ${ }^{2}$ \\ ${ }^{1}$ Gene Therapy Program, Fundacion para la Investigacion Medica Aplicada, CIMA, Universidad de Navarra, Instituto de Investigación Sanitaria de Navarra \\ (IdiSNA), Av. Pio XII 55, Pamplona 31008, Spain \\ ${ }^{2}$ Oncohematology Research Group, Navarrabiomed, Complejo Hospitalario de Navarra, Universidad Pública de Navarra, Instituto de Investigación Sanitaria de \\ Navarra (IdiSNA). Irunlarrea 3. 31008 Pamplona. Spain
}

\begin{abstract}
The influence of chemokines in tumor development is of special importance. In this work we will highlight several studies that revolve around IL- 8 and show the multiple facets that could have in the immune response. The influence of IL-8 is like an actor who has different roles in the same tumor movie.
\end{abstract}

\section{IL-8 levels correlate with tumor burden in preclinical models and in patients with cancer}

In several analyzes performed in the context of immunotherapy clinical trials we have observed that IL- 8 can serve as a serum biomarker for monitoring tumors [1]. We correlated IL-8 serum levels with baseline tumor burden and with treatment-induced changes in tumor burden, as well as with prognosis. The data clearly indicate that IL-8 is a potentially useful biomarker to monitor changes in tumor burden following anticancer therapy and has prognostic significance [1]. The monitoring of tumor burden is fundamental in the evaluation of the response to some therapies and treatment of patients with cancer. Actually, radiological images are the most used and reliable technology for this purpose, but they have important limitations when evaluating the tumor response especially to antiangiogenic drugs and immunotherapy. Each time they are discovering more serum tumor markers that are also useful in carcinomas of certain tissue origins. We found that serum concentrations of IL-8 accurately reflect the viability of tumor cells and correlate with tumor burden in a variety of solid human tumors. This change in values is possible to check at the beginning and after antitumor treatment, so it could be a useful platform for evaluating antiangiogenic and immunotherapeutic agents.

\section{IL-8 is a potentially useful biomarker to monitor} changes in tumor burden following anticancer therapy, and has prognostic significance

Substitute biomarkers of efficacy are needed for immunotherapies using anti-PD1/PD-L1, due to the existence of late responses and pseudo-progressions in the development of the disease [2]. To this end, we have studied the relationship between serum levels of IL- 8 and clinical performance of patients with advanced melanoma and patients with NSCLC during treatment with monotherapy using anti-PD-1 $\mathrm{mAbs}$, or in combination with anti-CTLA- $4 \mathrm{mAbs}$. We have found that changes in serum IL-8 levels have much potential for use in monitoring and predicting the clinical benefit of blocking the immune checkpoint in patients with melanoma and NSCLC $[3,4]$. We have found that in the same way that the tumor size correlated with the levels of IL-8 in the serum of the patients, it can be a very reliable biomarker of early tumor progression and check the changes that antitumor therapy is performing without prolonging a priori the duration of treatment.

DC become disoriented to subsequently follow IL-8 chemotactic gradients towards malignant or inflamed tissue

We have explored the effects of IL-8 in the biology of DC derived from human monocytes after exposure of this leukocyte population to recombinant IL-8 and with the use of an IL-8 neutralizing $\mathrm{mAb}$ [5]. The experimentation was performed evaluating different techniques: a) Experiments on immunodeficient mice xenografted with human colon carcinomas producing IL-8 and comparatively with cell lines that do not produce IL- 8 ; $b$ ) the stimulation of allogeneic T lymphocytes by DC was explored under the influence of IL-8; c) the chemotaxis of DC and neutrophils was measured by transwell migration assays.

As conclusions we can highlight that: a) Sera from mice xenografted with tumor contained increasing concentrations of IL-8 as the tumors were growing; b) the production of IL- 8 by carcinoma cells can be modulated by low doses of cyclophosphamide at the level of transcription; c) human DCs injected into xenografted HT29 or CaCo2 tumors are retained intratumorally in an IL-8 dependent manner; d) exposition of DC to IL-8 can desensitize for chemoattraction mediated by IL- 8 both in vitro and in vivo, indicating us that DC becomes

*Correspondence to: Carlos Alfaro Alegría, Oncohematology Research Group, Navarrabiomed, Complejo Hospitalario de Navarra, Universidad Pública de Navarra, Instituto de Investigación Sanitaria de Navarra (IdiSNA). Irunlarrea 3. 31008 Pamplona. Spain, E-mail: carlos.alfaro.alegria@navarra.es

Key words: Interleukin-8; tumor development; immune response

Received: September 15, 2018; Accepted: September 26, 2018; Published: September 28, 2018 
disoriented to subsequently follow chemotactic gradients to malignant or inflamed tissue; e) finally, IL-8 did not modify the ability of DC to stimulate $\mathrm{T}$ cells.

\section{IL-8 co-attracts polymorphonuclear cells (PMN) and $\mathrm{DC}$ at the same focus and antigens phagocytosed by short-lived PMNs can be in turn internalized and productively cross-presented by DC}

Another possible role of IL- 8 is the attraction of multiple lymphocyte populations to the same source of emission. It is especially important in the regulation of the immune response for tumor development and may even be responsible in part for the suppression of this antitumor response [6].

In this case we have verified that IL-8 is able to attract both DC and neutrophils to the same place, where they are in close contact [7]. This allows a transfer of material between the cells that subsequently can trigger an immune response that favors tumor development [7].

Our experiments make evident complex relationship between PMN and DC. Physiologically, the PMN are much more numerous than the DC and, therefore, could act as possible accumulators of antigens and microbial molecules for DC. DC can internalize the material present in the PMN and then modulate DC functions while transferring the antigens that PMN may carry. It is within the possibility that this phenomenon may occur in the same manner by endogenous DC and could take special importance in the immune response against the tumor. However, exactly how relevant are these functions for the overall physiology of the immune system still remains to be seen.

\section{IL-8 derived from tumors contributes to the chemotactic recruitment of MDSC and to their functional control}

Following the previous epigraph, we have explored the relevance of the IL-8 attraction influence towards possible suppressive populations that are found in the tumor microenvironment [8].

The suppressive myeloid cells (Myeloid-Derived Suppressor Cells, MDSC) constitute a heterogeneous population of immature cells composed of macrophages, granulocytes and other populations of myeloid origin in early stages of differentiation. They especially have an important immunosuppressive component of $\mathrm{T}$ cells in cancer patients, as well as being able to promote the expansion of regulatory $\mathrm{T}$ cells [9]. Currently, the factors capable of attracting this cellular subtype to the tumor microenvironment are poorly understood. We have verified in previous works [8] that IL-8 (CXCL8) is a chemokine produced by cancer cells and whose serum concentration correlates with the tumor burden of patients and with a poor prognosis of the disease. We have shown that IL- 8 produced by cancer cells attracts by chemotaxis to suppressive myeloid cells obtained from the peripheral blood of patients with advanced cancer and that this chemotactic activity can be interrupted pharmacologically in tests in mice. Surprisingly, it was also found that IL-8 activates granulocytic myeloid suppressor cells to produce the formation of extracellular neutrophil traps (Neutrophil Extracellular Traps, NETs). These mechanisms mediated by IL-8 could be relevant in the establishment of a tumor microenvironment that favors the attraction of leukocytes that help the tumor to evade the immune system. Definitely, IL-8 produced by tumors contributes to the chemotactic attraction of suppressive myeloid cells and their functional control [10].

\section{Conclusions}

We have observed the implication of IL- 8 as a biomarker in several tumors and as chemoattractant of human myeloid suppressor cells and neutrophils. Likewise, it is still early for its application as a biomarker since we must perform experimentation aimed at confirming these results in larger series of patients. Evenly it is very important to take into account the role of this cytokine on co-attraction and migration of dendritic cells and neutrophils. In conclusion, there could be a muchdefined axis where IL-8 plays a very important role in the recruitment of certain lymphocyte populations and tumor development.

\section{References}

1. Sanmamed MF, Carranza-Rua O, Alfaro C, Oñate C, Martín-Algarra S, et al. (2014) Serum interleukin- 8 reflects tumor burden and treatment response across malignancies of multiple tissue origins. Clin Cancer Res 20: 5697-707. [Crossref]

2. Buder-Bakhaya K, Hassel JC (2018) Biomarkers for Clinical Benefit of Immune Checkpoint Inhibitor Treatment-A Review From the Melanoma Perspective and Beyond. Front Immunol 9: 1474. [Crossref]

3. Sanmamed MF, Perez-Gracia JL, Schalper KA, Fusco JP, Gonzalez A, et al. (2017) Changes in serum interleukin-8 (IL-8) levels reflect and predict response to anti-PD-1 treatment in melanoma and non-small-cell lung cancer patients. Ann Oncol 28: 19881995. [Crossref]

4. Popovic A, Jaffee EM, Zaidi N (2018) Emerging strategies for combination checkpoint modulators in cancer immunotherapy. J Clin Invest 128: 3209-3218. [Crossref]

5. Alfaro C, Suárez N, Martínez-Forero I, Palazón A, Rouzaut A, et al. (2011) Carcinomaderived interleukin-8 disorients dendritic cell migration without impairing T-cell stimulation. PLoS One 6: e17922. [Crossref]

6. Karin N (2018) Chemokines and cancer: new immune checkpoints for cancer therapy Curr Opin Immunol 51: 140-145. [Crossref]

7. Alfaro C, Suarez N, Oñate C, Perez-Gracia JL, Martinez-Forero I, et al. (2011) Dendritic cells take up and present antigens from viable and apoptotic polymorphonuclear leukocytes. PLoS One 6: e29300. [Crossref]

8. Alfaro C, Teijeira A, Onate C, Pérez G, Sanmamed MF, et al. (2016) Tumor-Produced Interleukin-8 Attracts Human Myeloid-Derived Suppressor Cells and Elicits Extrusion of Neutrophil Extracellular Traps (NETs). Clin Cancer Res 22: 3924-36. [Crossref]

9. Fujimura T, Kambayashi Y, Aiba S (2012) Crosstalk between regulatory T cells (Tregs) and myeloid derived suppressor cells (MDSCs) during melanoma growth Oncoimmunology 1: 1433-1434. [Crossref]

10. Alfaro C, Sanmamed MF, Rodríguez-Ruiz ME, Teijeira Á, Onate C, et al. (2017) Interleukin-8 in cancer pathogenesis, treatment and follow-up. Cancer Treat Rev 60: 24-31. [Crossref]

Copyright: (C2018 González M. This is an open-access article distributed under the terms of the Creative Commons Attribution License, which permits unrestricted use, distribution, and reproduction in any medium, provided the original author and source are credited. 